suffocated (compared to SIDS) were more likely to be $\geq 8$ months old (OR 2.6, 95\% CI 1.5 to 4.7), found on their stomach (OR 1.8, $95 \%$ CI 1.3 to 2.6 ), in an adult bed (OR 2.3, 95\% CI 1.6 to 3.2 ) or couch (OR 2.8, 95\% CI 1.7 to 4.8). Compared to undetermined cause deaths, infants who suffocated were more likely to be found on their stomach (OR 1.7, 95\% CI 1.3 to 2.2), in an adult bed (OR $1.4,95 \%$ CI 1.0 to 1.9 ) or couch (OR $1.7,95 \%$ CI 1.1 to 2.5 ).

Significance/Contribution to the Field Injury circumstances and risk factors in infant suffocation deaths differ from SIDS and undetermined cause deaths. These findings offer clues for prevention.

\section{NOW I LAY YOU DOWN TO SLEEP: REDUCING SUFFOCATION RISK IN THE INFANT SLEEP ENVIRONMENT}

doi:10.1136/injuryprev-2012-040590d.8

${ }^{1} \mathrm{P}$ Schnitzer, ${ }^{2} \mathrm{~T}$ Covington, ${ }^{2} \mathrm{H}$ Dykstra. ${ }^{1}$ University of Missouri, USA; ${ }^{2}$ Michigan Public Health Institute, USA

Background Suffocation is the leading cause of injury death among infants in the USA. Infant suffocation mortality rates increased from 2.8 to 12.5 deaths/100 000 live births between 1984 and 2004 . This increase has coincided with a decrease in sudden infant death syndrome (SIDS) and an increase in infant mortality due to undetermined causes.

Aims/Objectives/Purpose Describe the circumstances of sleep-related infant deaths and compare the injury circumstances and risk factors of infant suffocation deaths to those diagnosed as SIDS and undetermined causes to inform epidemiology, prevention and policy.

Methods Infant sleep-related deaths that occurred from nine states between 2005 and 2008 were analysed. Logistic regression analysis was conducted comparing suffocation deaths to SIDS and undetermined cause deaths.

Results/Outcomes A total of 3136 sleep-related infant deaths were analysed: 939 (29.9\%) diagnosed as suffocation, 960 (30.6\%) as SIDS and 1237 (39.5\%) as undetermined cause. Infants who 